\title{
PLANAR $p$-ELASTIC CURVES AND RELATED GENERALIZED COMPLETE ELLIPTIC INTEGRALS
}

\author{
Kohtaro Watanabe
}

\begin{abstract}
Planar elastica problem is a classical but has broad connections with various fields, such as elliptic functions, differential geometry, soliton theory, material mechanics, etc. This paper regards classical elastica as a theory corresponding to Lebesgue $L^{2}$ case, and extends it to $L^{p}$ cases. For the sake of the effect of $p$-Laplacian, novel curious solutions appear especially for cases $p>2$. These solutions never appear in $1<p \leq 2$ cases and we call them flat-core solutions according to Takeuchi $[6,7]$.
\end{abstract}

\section{Introduction}

Let $C$ be a plane curve with length $L, s$ an arc-length parameter and $\kappa(s)$ its curvature. Further, we fix an orthogonal coordinate system $(x, y)$ in the plane, and $\theta(s)$ be an angle between a tangent $(d x(s) / d s, d y(s) / d s)$ at the point $(x(s), y(s)) \in C$ and the positive $x$-axis. It is well-known that classical variational problem called "elastica", see for example Antman [1], Truesdell [8], minimizes the total squared curvature (elastic energy) of $C$ :

$$
J(\theta)=\frac{1}{2} \int_{0}^{L}\left(\theta_{s}(s)\right)^{2} d s=\frac{1}{2} \int_{0}^{L} \kappa^{2}(s) d s,
$$

subject to

$$
\begin{gathered}
\theta \in\left\{\theta \in C^{2}[0, L] \mid \int_{0}^{L} \cos \theta(s) d s=a, \int_{0}^{L} \sin \theta(s) d s=0\right. \\
\theta(0)=0, \theta(L)=2 n \pi(n \in \mathbf{N} \cup\{0\})\}
\end{gathered}
$$

2000 Mathematics Subject Classification. Primary 15A15, Secondary 15A09, 15A23.

Key words and phrases. elastica, $p$-Laplacian, generalized complete elliptic integrals, flat-core solution.

This work is partially supported by the Grant-in-Aid for Scientific Research (C) (No. 24540199) from Japan Society for the Promotion of Science.

Received November 5, 2013; revised December 10, 2013. 
where $a$ runs over the range $(-L \leq a \leq L)$. To show the existence of the minimizer, it is convenient to extend the definition domain of $\theta$ to some subset of Sobolev space $W^{1,2}(0, L)$ :

$$
\begin{gathered}
W(2, a, n):=\left\{\theta \in W^{1,2}(0, L) \mid \int_{0}^{L} \cos \theta(s) d s=a, \int_{0}^{L} \sin \theta(s) d s=0,\right. \\
\theta(0)=0, \theta(L)=2 n \pi\} .
\end{gathered}
$$

The stationary curve, i.e. the solution of the Euler-Lagrange equation for the functional $J$ is called elastic curves and their structures are well known: see Fig. 1 (Fig. 1 is the same one as Fig. 1.7 of Koiso [3]). As we see form this figure, elastic curves are intrinsically periodic. For this reason, we have imposed the condition $\theta(0)=0, \theta(L)=2 n \pi(n \in \mathbf{N} \cup\{0\})$. As a natural extension, one may think of area constraint (if curves are restricted to be closed) as an additional constraint condition, for this aspect see; $[10,4]$. This paper regards classical elastica problem as $L^{2}$ case and extends it to $L^{p}$ problem, that is:

Minimize

$$
J(\theta)=\frac{1}{p} \int_{0}^{L}\left|\theta_{s}(s)\right|^{p} d s=\frac{1}{p} \int_{0}^{L}|\kappa(s)|^{p} d s .
$$

Subject to $\theta \in W(p, a, n)$ :

$$
\begin{gathered}
W(p, a, n):=\left\{\theta \in W^{1, p}(0, L) \mid \int_{0}^{L} \cos \theta(s) d s=a, \int_{0}^{L} \sin \theta(s) d s=0,\right. \\
\theta(0)=0, \theta(L)=2 n \pi\},
\end{gathered}
$$

where $a$ and $n$ are as before. Although the existence of the minimizer can be readily shown with this setting; see Appendix, we are much more concerned with the detailed relation between the solutions of the Euler-Lagrange equation and the shapes of stationary curves. So, let us introduce the Euler-Lagrange equation for this case concretely. Since

$$
x(L)=\int_{0}^{L} \cos \theta(s) d s=a, \quad y(L)=\int_{0}^{L} \sin \theta(s) d s=0,
$$

if $\theta$ is stationary, there exist Lagrange multipliers $\lambda_{0}, \lambda_{1}, \lambda_{2} \in \mathbf{R}$, such that for arbitrary $\varphi \in W^{1, p}(0, L)$ satisfying $\varphi(0)=\varphi(L)=0$ (since the values of $\theta(0)$ and $\theta(L)$ are fixed), it holds that

$$
\lambda_{0} \int_{0}^{L}\left|\theta_{s}\right|^{p-2} \theta_{s} \varphi_{s}(s) d s-\lambda_{1} \int_{0}^{L} \sin \theta(s) \varphi(s) d s-\lambda_{2} \int_{0}^{L} \cos \theta(s) \varphi(s) d s=0 .
$$




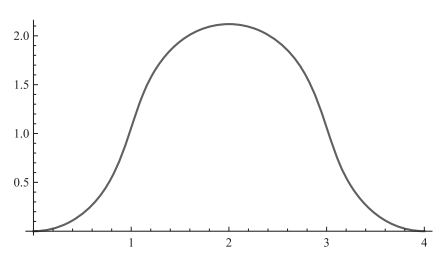

$\mathrm{a}=4.0, \mathrm{n}=0, \mathrm{~m}=1$

Case (ii)

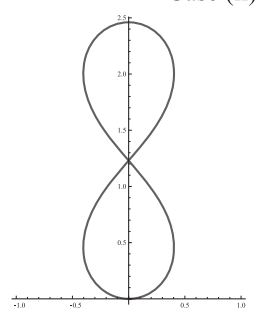

$\mathrm{a}=0, \mathrm{n}=0, \mathrm{~m}=1$

Case (vi)

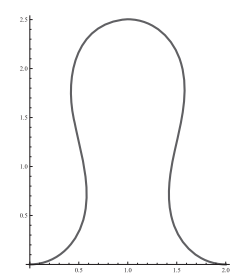

$\mathrm{a}=2.0, \mathrm{n}=0, \mathrm{~m}=1$

Case (ii)

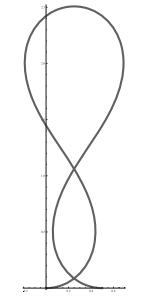

$\mathrm{a}=0.5, \mathrm{n}=0, \mathrm{~m}=1$

Case (ii)

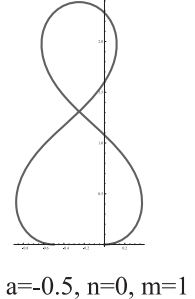

Case (ii)

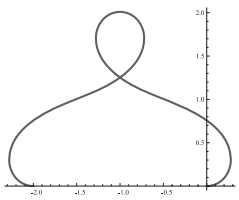

$a=-2.0, n=0, m=1$

Case (ii)

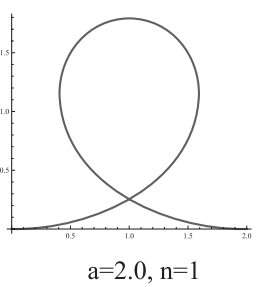

Case (iv)

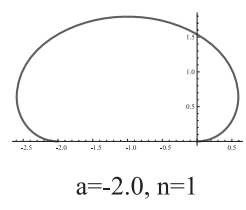

Case (iii)

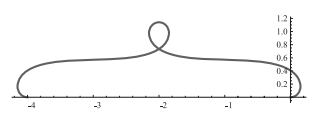

$\mathrm{a}=-4.0, \mathrm{n}=0, \mathrm{~m}=1$

Case (ii)

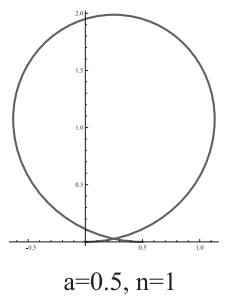

Case (iv)

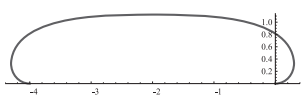

$\mathrm{a}=-4.0, \mathrm{n}=1$

Case (iii)

FIGURE 1. Elastic curves of various $a, n$ and $m$ for $L=2 \pi$, where the parameter $m$ means the number of shape repetition (see Theorem 1). 
If $\lambda_{0}=0, \theta \equiv 0$ holds, and hence the stationary curve is $(x, y)=(s, 0)(0 \leq s \leq L)$. Thus, in the following we assume $\lambda_{0}=1$, and consider the equation:

$$
\begin{aligned}
& \left(\left|\theta_{s}\right|^{p-2} \theta_{s}\right)_{s}=-\lambda_{1} \sin \theta-\lambda_{2} \cos \theta, \\
& \theta(0)=0, \quad \theta(L)=2 n \pi, \quad(n \in \mathbf{N} \cup\{0\}), \\
& \int_{0}^{L} \cos \theta(s) d s=a, \quad(-L \leq a \leq L), \\
& \int_{0}^{L} \sin \theta(s) d s=0 .
\end{aligned}
$$

We call a solution which satisfies (4)-(7), p-elastic curve. Clearly, p-elastic curves are stationary because admissible functions $\varphi$ satisfy

$$
\int_{0}^{L} \sin \theta(s) \varphi(s) d s=0, \quad \int_{0}^{L} \cos \theta(s) \varphi(s) d s=0 .
$$

Since the equation (4) includes $p$-Laplacian term, as is seen in Takeuchi $[6,7]$, the value $p=2$ divides qualitative behavior of the solution. Now, putting $\lambda_{1}=$ $R \cos \alpha, \lambda_{2}=R \sin \alpha$ where $R=\sqrt{\lambda_{1}^{2}+\lambda_{2}^{2}}$, we have from (4),

$$
\left(\left|\theta_{s}\right|^{p-2} \theta_{s}\right)_{s}=-R \sin (\theta+\alpha) .
$$

Further, multiplying $\theta_{s}$ both sides of (9), we obtain $L^{p}$ extension of energy conservation law:

$$
\frac{p-1}{p}\left|\theta_{s}\right|^{p}=E+R \cos (\theta+\alpha)
$$

where $E$ is a constant (corresponding to a total energy). Here, $E, R$ and $\alpha$ must be taken to satisfy (5), (6) and (7). We can assume without loss of generality, $\alpha=0$. Since if solutions of (10) satisfying (5), (6) and (7) exists for $E=E_{0}$, $R=R_{0}, \alpha=\alpha_{0}$, it can be obtained by suitable parallel translation and rotating $-\alpha_{0}(\mathrm{rad})$ the curve generated for $E=E_{0}, R=R_{0}, \alpha=0$. Thus, we analyze

$$
\frac{p-1}{p}\left|\theta_{s}\right|^{p}=E+R \cos \theta
$$

for the following five cases:

$$
\begin{cases}(\mathrm{I}) & R=0 \\ (\mathrm{II}) & E=-R \\ (\mathrm{III}) & -R<E<R \\ (\mathrm{IV}) & E=R \\ (\mathrm{~V}) & R<E\end{cases}
$$


where, for cases (II) to $(\mathrm{V})$, we assume $R>0$. In the next section, main results are stated, and in Section 3, we prove the results of Section 2, in accordance with above cases.

\section{Main results}

To state the results, we introduce some notations which are generalization of complete elliptic integrals:

\section{Definition 1.}

$$
\begin{aligned}
& K_{1, p}(q)=\int_{0}^{\pi / 2} \frac{(\cos \phi)^{1-2 / p}}{\sqrt{1-q^{2} \sin ^{2} \phi}} d \phi \\
& E_{1, p}(q)=\int_{0}^{\pi / 2} \sqrt{1-q^{2} \sin ^{2} \phi}(\cos \phi)^{1-2 / p} d \phi \\
& K_{2, p}(q)=\int_{0}^{\pi / 2} \frac{1}{\sqrt[p]{1-q^{2} \sin ^{2} \phi}} d \phi \\
& E_{2, p}(q)=\int_{0}^{\pi / 2} \sqrt[p]{1-q^{2} \sin ^{2} \phi} d \phi
\end{aligned}
$$

When $p=2$, we see that these integrals coincide with complete elliptic integrals of first and second kinds.

Remark 1. In [7, p. 89], a generalization of complete elliptic integral is given by

$$
K_{p, m, r}(q)=\int_{0}^{1} \frac{d s}{\sqrt[p]{1-s^{m}} \sqrt[r]{1-q^{m} s^{m}}}=\int_{0}^{\pi / 2} \frac{\cos \phi}{\sqrt[p]{1-\sin ^{m} \phi} \sqrt[r]{1-q^{m} \sin ^{m} \phi}} d \phi
$$

where $m>1$ is a parameter. So, if we take $m=2, r=2$ or $p=2, m=2, r=p$ in (17), we obtain $K_{1, p}(q), K_{2, p}(q)$ respectively. Nevertheless, we would like to use the notation in Definition 1 for the simplicity.

For $1<p \leq 2$, we have the following result:

THEOREM 1. Let $1<p \leq 2$. Then, the solutions of (4)-(7) i.e. p-elastic curves $(x, y)$ exist such that followings hold:

(i) The case $a= \pm L$, and $n=0$.

$$
x(s)= \pm s, \quad y(s)=0, \quad(0 \leq s \leq L) .
$$

(ii) The case $-L<a<L(a \neq 0)$ and $n=0$. 
(18) $x(\phi)=\frac{L}{4 m} K_{1, p}(q)^{-1}\left(2 \int_{0}^{\phi} \sqrt{1-q^{2} \sin ^{2} \varphi}|\cos \varphi|^{1-2 / p} d \varphi\right.$

$$
\begin{gathered}
\left.-\int_{0}^{\phi} \frac{|\cos \varphi|^{1-2 / p}}{\sqrt{1-q^{2} \sin ^{2} \varphi}} d \varphi\right) \\
y(\phi)= \pm \frac{L}{4 m} K_{1, p}(q)^{-1} \int_{0}^{\phi} \frac{\sin \left\{2 \sin ^{-1}(q \sin \varphi)\right\}|\cos \varphi|^{1-2 / p}}{\sqrt{1-q^{2} \sin ^{2} \varphi}} d \varphi
\end{gathered}
$$

where $m \in \mathbf{N}, 0 \leq \phi \leq 2 m \pi$ and $q$ is a unique solution of the equation

$$
L K_{1, p}(q)^{-1}\left(2 E_{1, p}(q)-K_{1, p}(q)\right)=a .
$$

We note that the p-elastic curves generated by q correspond to case (III) of (12).

(iii) The case $-L<a<0$ and $n \in \mathbf{N}$.

$$
\begin{aligned}
& x(\phi)=\frac{L}{2 n}\left(K_{2, p}(q)\right)^{-1} \int_{0}^{\phi} \frac{\cos 2 \varphi}{\sqrt[p]{1-q^{2} \sin ^{2} \varphi}} d \varphi \\
& y(\phi)=\frac{L}{2 n}\left(K_{2, p}(q)\right)^{-1} \int_{0}^{\phi} \frac{\sin 2 \varphi}{\sqrt[p]{1-q^{2} \sin ^{2} \varphi}} d \varphi,
\end{aligned}
$$

where $0 \leq \phi \leq n \pi$, and $q$ is a unique solution of the equation

$$
L\left(K_{2, p}(q)\right)^{-1}\left(\frac{q^{2}-2}{q^{2}} K_{2, p}(q)+\frac{2}{q^{2}} E_{2, p /(p-1)}(q)\right)=a .
$$

(iv) The case $0<a<L$ and $n \in \mathbf{N}$.

$$
\begin{aligned}
& x(\phi)=\frac{L}{2 n}\left(K_{2, p}(q)\right)^{-1} \int_{0}^{\phi} \frac{\cos 2 \varphi}{\sqrt[p]{1-q^{2} \cos ^{2} \varphi}} d \varphi \\
& y(\phi)=\frac{L}{2 n}\left(K_{2, p}(q)\right)^{-1} \int_{0}^{\phi} \frac{\sin 2 \varphi}{\sqrt[p]{1-q^{2} \cos ^{2} \varphi}} d \varphi,
\end{aligned}
$$

where $0 \leq \phi \leq n \pi$, and $q$ is a unique solution of the equation

$$
-L\left(K_{2, p}(q)\right)^{-1}\left(\frac{q^{2}-2}{q^{2}} K_{2, p}(q)+\frac{2}{q^{2}} E_{2, p /(p-1)}(q)\right)=a .
$$

For cases (iii) and (iv), we note that the p-elastic curves generated by $q$ correspond to case (V) of (12). 
(v) The case $a=0$ and $n \in \mathbf{N}$.

For this case, locus of $(x, y)$ is a circle of radius $L / 2 n \pi$.

(vi) The case $a=0$ and $n=0$.

$$
\begin{gathered}
x(\phi)=\frac{L}{4 m} K_{1, p}(q)^{-1}\left(2 \int_{\alpha}^{\phi+\alpha} \sqrt{1-q^{2} \sin ^{2} \varphi}|\cos \varphi|^{1-2 / p} d \varphi\right. \\
\left.-\int_{\alpha}^{\phi+\alpha} \frac{|\cos \varphi|^{1-2 / p}}{\sqrt{1-q^{2} \sin ^{2} \varphi}} d \varphi\right) \\
y(\phi)= \pm \frac{L}{4 m} K_{1, p}(q)^{-1} \int_{\alpha}^{\phi+\alpha} \frac{\sin \left\{2 \sin ^{-1}(q \sin \varphi)\right\}|\cos \varphi|^{1-2 / p}}{\sqrt{1-q^{2} \sin ^{2} \varphi}} d \varphi
\end{gathered}
$$

where $m \in \mathbf{N}, 0 \leq \phi \leq 2 m \pi, \alpha$ is an arbitrary angle satisfying $0 \leq \alpha<2 \pi$ and $q$ is a unique solution of the equation

$$
L K_{1, p}(q)^{-1}\left(2 E_{1, p}(q)-K_{1, p}(q)\right)=0 .
$$

Figure 2 shows examples of $p$-elastic curves for $p=1.2$ and $L=2 \pi$. For the case $p>2$, we have the following theorem. In this case, thanks to the existence of $p$-Laplacian term, novel curious solutions called flat-core solutions appear. We note our flat-core solutions are essentially the same as the one introduced by Takeuchi [6, 7].

THEOREM 2. Let $p>2$. Then, the solutions of (4)-(7) i.e. p-elastic curves $(x, y)$ exist such that followings hold:

(i) The case $a= \pm L$, and $n=0$.

$$
x(s)= \pm s, \quad y(s)=0, \quad(0 \leq s \leq L) .
$$

(ii) The case $-L /(p-1)<a<L(a \neq 0)$ and $n=0$.

For this case, $(x, y)$ satisfies (18), where $q$ is the unique solution of (19). As Theorem 1, the p-elastic curves generated by q correspond to case (III) of (12).

(iii) The case $-L /(p-1)<a<0$ and $n \in \mathbf{N}$.

For this case, $(x, y)$ satisfies (20), where $q$ is the unique solution of (21). As Theorem 1, the p-elastic curves generated by $q$ correspond to case (V) of (12).

(iv) The case $0<a<L /(p-1)$ and $n \in \mathbf{N}$.

For this case, $(x, y)$ satisfies (22), where $q$ is the unique solution of (23). As Theorem 1, the p-elastic curves generated by q correspond to case (V) of (12).

(v) The case $a=0$ and $n \in \mathbf{N}$.

For this case, locus of $(x, y)$ is a circle of radius $L / 2 n \pi$. 


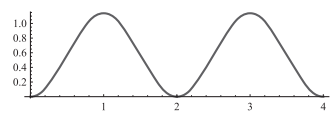

$\mathrm{a}=4.0, \mathrm{n}=0, \mathrm{~m}=2$

Case (ii)
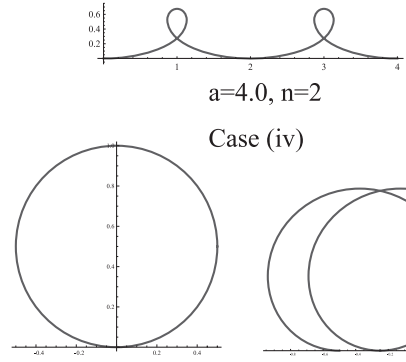

$\mathrm{a}=0, \mathrm{n}=2$

Case (v)

$\mathrm{a}=0, \mathrm{n}=0, \mathrm{~m}=2$

Case (ii)

Case (iii)

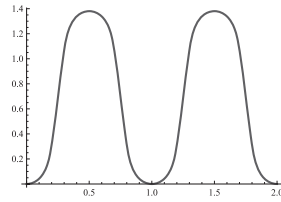

$\mathrm{a}=2.0, \mathrm{n}=0, \mathrm{~m}=2$

Case (ii)

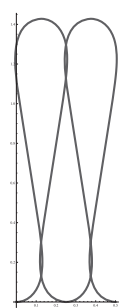

$\mathrm{a}=0.5, \mathrm{n}=0, \mathrm{~m}=2$

Case (ii)
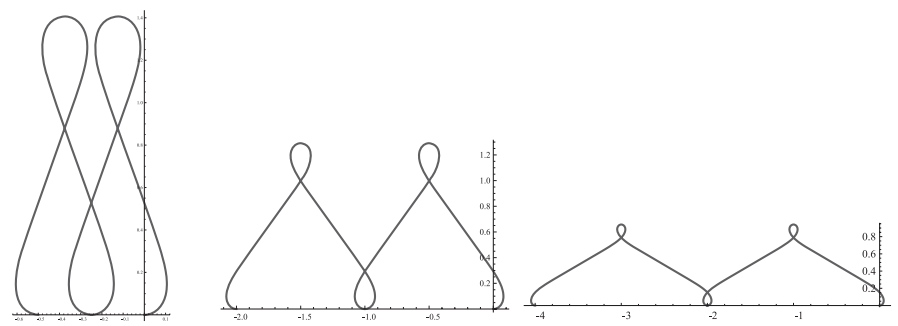

$\mathrm{a}=-0.5, \mathrm{n}=0, \mathrm{~m}=2$

$\mathrm{a}=-2.0, \mathrm{n}=0, \mathrm{~m}=2$

Case (ii)

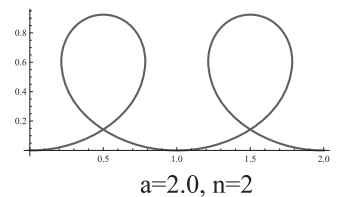

Case (iv)

Case (ii)

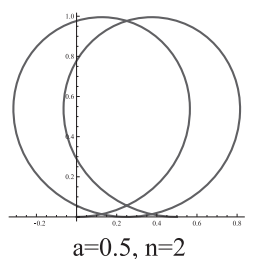

Case (iv)

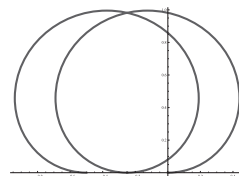

$\mathrm{a}=-0.5, \mathrm{n}=2$

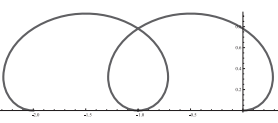

$\mathrm{a}=-2.0, \mathrm{n}=2$

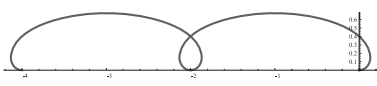

$\mathrm{a}=-4.0, \mathrm{n}=2$

Case (iii)

FIGURE 2. $p$-elastic curves for $p=1.2, L=2 \pi$.

(vi) The case $a=0$ and $n=0$.

For this case, $(x, y)$ satisfies (24), where $q$ is the unique solution of (25).

To describe the existence result of flat core solutions, we introduce following definition. 

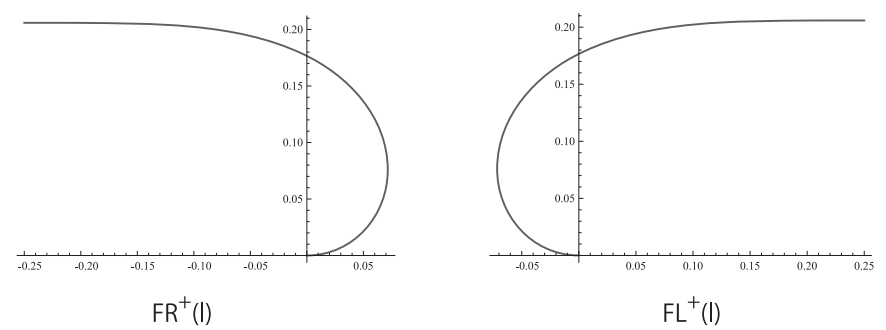

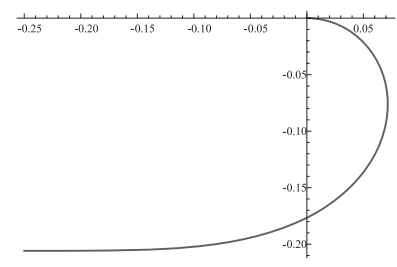

$\mathrm{FR}^{-}(\mathrm{l})$

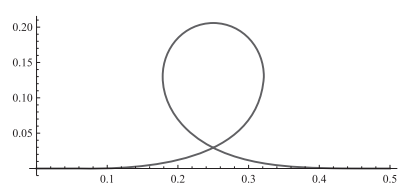

$\mathrm{F}^{+}(\mathrm{l})$

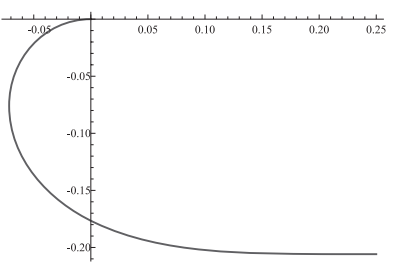

$\mathrm{FL}^{-}(\mathrm{l})$

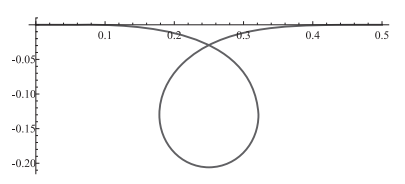

$\mathrm{F}^{-}(\mathrm{I})$

Figure 3. $F R^{+}(l), F L^{+}(l), F R^{-}(l), F L^{-}(l), F^{+}(l)$, and $F^{-}(l)$, when $l=1$.

Definition 2. We denote by $L(l)$ a line segment of length $l$ parallel to $x$ axis. Moreover, $F R^{+}(l), F L^{+}(l), F R^{-}(l), F L^{-}(l), F^{+}(l), F^{-}(l)$ are curves defined by the following expressions. Here, $(x(\phi), y(\phi))(0 \leq \phi \leq \pi / 2)$ denote the locus of the curves; see Figure 3.

$$
F R^{ \pm}(l):\left\{\begin{array}{l}
x(\phi)=\frac{l \Gamma\left(\frac{p-1}{p}\right)}{\sqrt{\pi} \Gamma\left(\frac{p-2}{2 p}\right)} \int_{0}^{\phi} \frac{\cos 2 \varphi}{(\cos \varphi)^{2 / p}} d \varphi \\
y(\phi)= \pm \frac{l \Gamma\left(\frac{p-1}{p}\right)}{\sqrt{\pi} \Gamma\left(\frac{p-2}{2 p}\right)} \int_{0}^{\phi} \frac{\sin 2 \varphi}{(\cos \varphi)^{2 / p}} d \varphi
\end{array}\right.
$$




$$
F L^{ \pm}(l):\left\{\begin{array}{l}
x(\phi)=-\frac{l \Gamma\left(\frac{p-1}{p}\right)}{\sqrt{\pi} \Gamma\left(\frac{p-2}{2 p}\right)} \int_{0}^{\phi} \frac{\cos 2 \varphi}{(\cos \varphi)^{2 / p}} d \varphi \\
y(\phi)= \pm \frac{l \Gamma\left(\frac{p-1}{p}\right)}{\sqrt{\pi} \Gamma\left(\frac{p-2}{2 p}\right)} \int_{0}^{\phi} \frac{\sin 2 \varphi}{(\cos \varphi)^{2 / p}} d \varphi \\
F^{ \pm}(l):\left\{\begin{array}{l}
x(\phi)=\frac{l \Gamma\left(\frac{p-1}{p}\right)}{\sqrt{\pi} \Gamma\left(\frac{p-2}{2 p}\right)} \int_{0}^{\phi} \frac{\cos 2 \varphi}{(\sin \varphi)^{2 / p}} d \varphi \\
y(\phi)= \pm \frac{l \Gamma\left(\frac{p-1}{p}\right)}{\sqrt{\pi} \Gamma\left(\frac{p-2}{2 p}\right)} \int_{0}^{\phi} \frac{\sin 2 \varphi}{(\sin \varphi)^{2 / p}} d \varphi
\end{array}\right.
\end{array}\right.
$$

In addition, we introduce following numbers: $n_{+} \in \mathbf{N}, n_{-}, n_{l} \in \mathbf{N} \cup\{0\}$, $L_{0}>0, L_{i}>0\left(1 \leq i \leq n_{l}\right)$. With these notions, we obtain the structure theorem of flat core solutions.

THEOREM 3. Let $p>2$. Then, the solutions of (4)-(7) i.e. p-elastic curves $(x, y)$ are as cases (i)-(vi) of Theorem 2. In addition, we have the following case.

(vii) (Flat Core Solution Case I) The case $L /(p-1) \leq a<L$ and $n \in$ $\mathbf{N} \cup\{0\}$.

Let $n_{+}, n_{-}, n_{0}$, be non-negative integers, $L_{0}>0, L_{i} \geq 0\left(1 \leq i \leq n_{0}\right)$ satisfying $n=n_{+}-n_{-}$and $L=L_{0}\left(n_{+}+n_{-}\right)+\sum_{i=1}^{n_{0}} L_{i}$ (if $n_{0}=0$, we assume $\left.L=L_{0}\left(n_{+}+n_{-}\right)\right)$. Then, p-elastic curves consists of $n_{+}$piece of $F^{+}\left(L_{0}\right), n_{-}$piece of $F^{-}\left(L_{0}\right)$ and $n_{0}$ piece of line segments $L\left(L_{i}\right)$ $\left(1 \leq i \leq n_{0}\right)$ (if $n_{0}=0$, it means there is no line segments) joined each other in arbitrary order, but satisfying both end points are on $(0,0)$ and $(a, 0)$; see Figure 4. We express these solutions with $F^{+}\left(L_{0}\right), F^{-}\left(L_{0}\right)$ and $\left\{L\left(L_{i}\right)\right\} \quad\left(1 \leq i \leq n_{0}\right)$, in accordance with joined order from left to right.

(viii) (Flat Core Solution Case II-1) The case $-L<a \leq-L /(p-1)$ and $n \in \mathbf{N} \cup\{0\}$.

Let $n_{+}, n_{-}, n_{0}, L_{0}>0, L_{i} \geq 0\left(1 \leq i \leq n_{0}\right)$ are as case (vii) and satisfying $n=n_{+}-n_{-}+1, L=L_{0}\left(n_{+}+n_{-}+1\right)+\sum_{i=1}^{n_{0}} L_{i}$ (if $n_{0}=0$, we assume $\left.L=L_{0}\left(n_{+}+n_{-}+1\right)\right)$. Then, p-elastic curves consists of $F L^{+}\left(L_{0}\right), F R^{+}\left(L_{0}\right), n_{+}$piece of $F^{-}\left(L_{0}\right), n_{-}$piece of $F^{+}\left(L_{0}\right)$ and $n_{0}$ piece of line segments $L\left(L_{i}\right)\left(1 \leq i \leq n_{0}\right)$ joined each other in arbitrary 


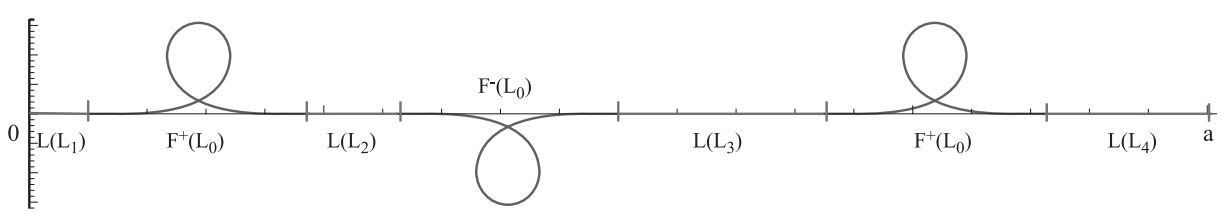

$$
\mathrm{L}\left(\mathrm{L}_{1}\right) \mathrm{F}^{+}\left(\mathrm{L}_{0}\right) \mathrm{L}\left(\mathrm{L}_{2}\right) \mathrm{F}^{-}\left(\mathrm{L}_{0}\right) \mathrm{L}\left(\mathrm{L}_{3}\right) \mathrm{F}^{+}\left(\mathrm{L}_{0}\right) \mathrm{L}\left(\mathrm{L}_{4}\right)
$$

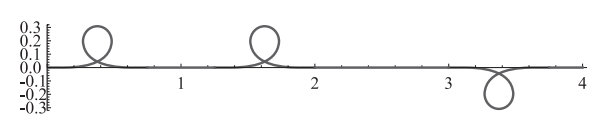

(i) $\mathrm{F}^{+}(1.5) \mathrm{L}(0.5) \mathrm{F}^{+}(1.5) \mathrm{L}(1) \mathrm{F}^{-}(1.5) \mathrm{L}(0.28)$ $\mathrm{a}=4.033, \mathrm{p}=3, \mathrm{n}=1$

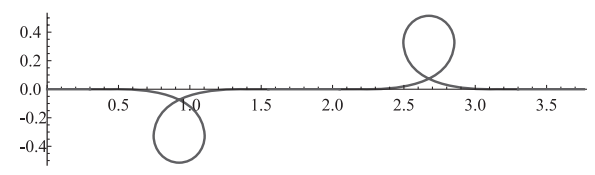

(iii) $\mathrm{L}(0.3) \mathrm{F}^{-}(2.5) \mathrm{L}(0.5) \mathrm{F}^{+}(2.5) \mathrm{L}(0.48)$

$\mathrm{a}=3.783, \mathrm{p}=3, \mathrm{n}=0$

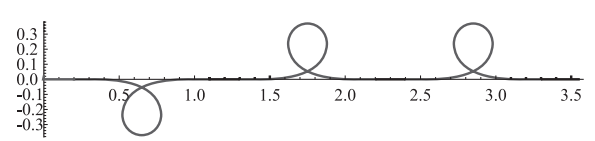

(ii) $\mathrm{L}(0.2) \mathrm{F}^{-}(1.8) \mathrm{L}(0.2) \mathrm{F}^{+}(1.8) \mathrm{L}(0.2) \mathrm{F}^{+}(1.8) \mathrm{L}(0.28)$ $\mathrm{a}=3.583, \mathrm{p}=3, \mathrm{n}=1$

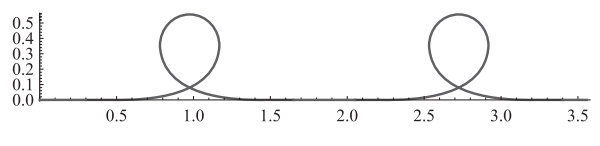

(iv) $\mathrm{L}(0.3) \mathrm{F}^{+}(2.7) \mathrm{L}(0.4) \mathrm{F}^{+}(2.7) \mathrm{L}(0.18)$ $\mathrm{a}=3.583, \mathrm{p}=3, \mathrm{n}=2$

FIGURE 4. Flat core solutions of the case (vii) of Theorem 3.

order, but satisfying both ends are $\mathrm{FL}^{+}\left(L_{0}\right)$ and $F R^{+}\left(L_{0}\right)$ and end points of them are on $(0,0)$ and $(a, 0)$ respectively; see Figure 5-(i). We express these solutions as in the case (vii).

(ix) (Flat Core Solution Case II-2) In the case $-L<a \leq-L /(p-1)$ and $n \in \mathbf{N} \cup\{0\}$.

Let $n_{+}, n_{-}, n_{0}, L_{0}, L_{i}\left(1 \leq i \leq n_{0}\right)$ are as case (vii) and satisfying $n=n_{+}-n_{-}-1, \quad L=L_{0}\left(n_{+}+n_{-}+1\right)+\sum_{i=1}^{n_{0}} L_{i} \quad$ (if $n_{0}=0$, we fix $\left.L=L_{0}\left(n_{+}+n_{-}+1\right)\right)$. Then, p-elastic curves consists of $F L^{-}\left(L_{0}\right)$, $F R^{-}\left(L_{0}\right), n_{+}$piece of $F^{-}\left(L_{0}\right), n_{-}$piece of $F^{+}\left(L_{0}\right)$ and $n_{0}$ piece of line segments $L\left(L_{i}\right)\left(1 \leq i \leq n_{0}\right)$ joined each other in arbitrary order, but satisfying both ends are $F L^{-}\left(L_{0}\right)$ and $F R^{-}\left(L_{0}\right)$ and end points of them are on $(0,0)$ and $(a, 0)$ respectively; see Figure 5-(ii). We express these solutions as in the case (vii).

\section{Proof of theorems}

We investigate cases (I) to $(\mathrm{V})$ of $(12)$ in detail. We note that for the proof of theorems, the argument of Yanamoto [9] was helpful. 


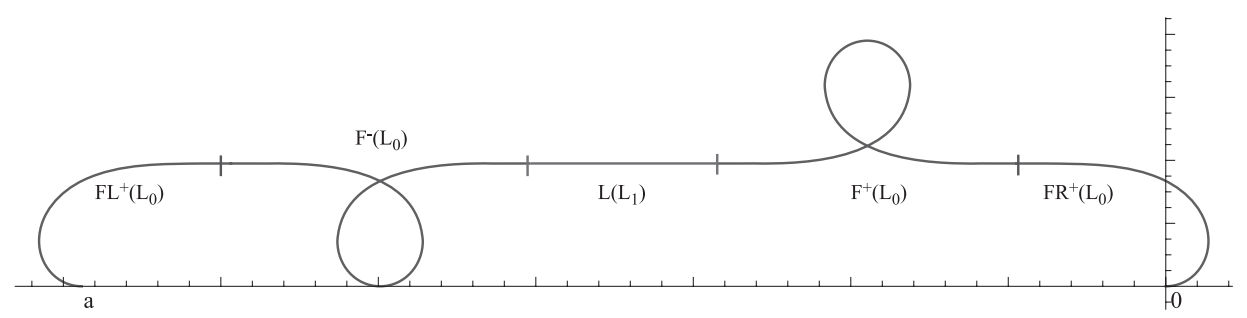

(i) $\mathrm{FL}^{+}\left(\mathrm{L}_{0}\right) \mathrm{F}^{-}\left(\mathrm{L}_{0}\right) \mathrm{L}\left(\mathrm{L}_{1}\right) \mathrm{F}^{+}\left(\mathrm{L}_{0}\right) \mathrm{FR}^{+}\left(\mathrm{L}_{0}\right)$

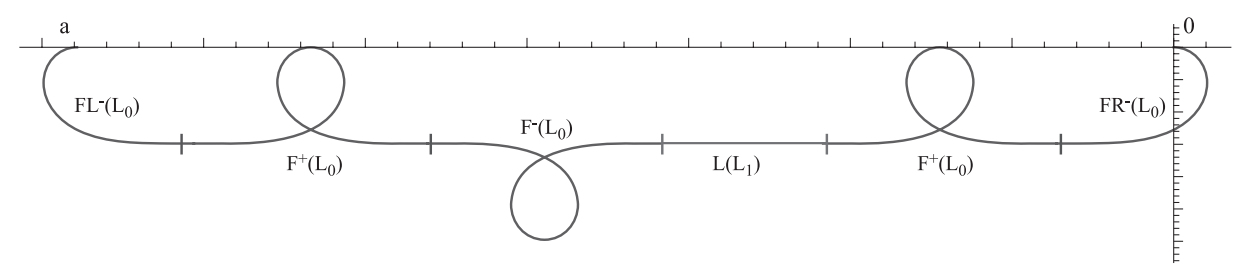

(ii) $\quad \mathrm{FL}^{-}\left(\mathrm{L}_{0}\right) \mathrm{F}^{+}\left(\mathrm{L}_{0}\right) \mathrm{F}^{-}\left(\mathrm{L}_{0}\right) \mathrm{L}\left(\mathrm{L}_{1}\right) \mathrm{F}^{+}\left(\mathrm{L}_{0}\right) \mathrm{FR}^{-}\left(\mathrm{L}_{0}\right)$

FIGURE 5. Flat core solutions of the case (viii) and the case (ix) of Theorem 3.

The case (I) of (12).

In this case, the solution of (11) is $\theta_{s} \equiv$ Constant. This applies to cases (i) and (v) of Theorem 1 and 2 .

The case (II) of (12).

In this case, the solution of (11) is $\theta \equiv 0$. This applies to cases (i) of Theorem 1 and 2 .

The case (III) of (12).

From (11), we have

$$
\frac{p-1}{p}\left|\theta_{s}\right|^{p}=E+R \cos \theta=2 R\left(\frac{E+R}{2 R}-\sin ^{2} \frac{\theta}{2}\right) .
$$

Putting $q^{2}=(E+R) / 2 R$, and noting it must be $\theta(0)=\theta(L)=0$ in this case, we have

$$
\theta_{s}(s)=\left(\frac{2 p R}{p-1}\right)^{1 / p} \sqrt[p]{q^{2}-\sin ^{2} \frac{\theta}{2}}, \quad\left(0 \leq s \leq \frac{L}{4 m}\right)
$$

where $m$ is a positive integer. Thus, we obtain

$$
s=\left(\frac{p-1}{2 p R}\right)^{1 / p} \int_{0}^{\theta} \frac{d \theta}{\sqrt[p]{q^{2}-\sin ^{2} \frac{\theta}{2}}}, \quad\left(0 \leq \theta \leq 2 \sin ^{-1} q\right) .
$$


It is convenient to introduce the new variable $\phi$ satisfying

$$
\sin \frac{\theta}{2}=q \sin \phi
$$

We note that as $\theta$ varies from 0 to $2 \sin ^{-1} q, \phi$ does from 0 to $\pi / 2$. Using $\phi$, we can see that $s$ can be expressed as

$$
s=2 q^{1-2 / p}\left(\frac{p-1}{2 p R}\right)^{1 / p} \int_{0}^{\phi} \frac{(\cos \phi)^{1-2 / p}}{\sqrt{1-q^{2} \sin ^{2} \phi}} d \phi, \quad\left(0 \leq \phi \leq \frac{\pi}{2}\right) .
$$

Moreover, from the symmetry of $\theta$ we can extend the definition domain of $s=s(\phi)$ from $[0, \pi / 2]$ to $[0,2 \pi]$ as

$$
s=2 q^{1-2 / p}\left(\frac{p-1}{2 p R}\right)^{1 / p} \int_{0}^{\phi} \frac{|\cos \phi|^{1-2 / p}}{\sqrt{1-q^{2} \sin ^{2} \phi}} d \phi, \quad(0 \leq \phi \leq 2 \pi) .
$$

From (32) and the relation $\phi(L / 4 m)=\pi / 2$, it holds that

$$
\frac{L}{4 m}=2 q^{1-2 / p}\left(\frac{p-1}{2 p R}\right)^{1 / p} \int_{0}^{\pi / 2} \frac{(\cos \phi)^{1-2 / p}}{\sqrt{1-q^{2} \sin ^{2} \phi}} d \phi .
$$

Thus $R$ can be expressed as a function of $q$ :

$$
R(q)=R=\frac{2^{3 p-1} m^{p} q^{p-2}(p-1)}{p L^{p}}\left(\int_{0}^{\pi / 2} \frac{(\cos \phi)^{1-2 / p}}{\sqrt{1-q^{2} \sin ^{2} \phi}} d \phi\right)^{p} .
$$

Applying (33), we can express $(x(L), y(L))$ as followings:

Lemma 1.

$$
\begin{aligned}
& x(L)=\int_{0}^{L} \cos \theta(s) d s=L K_{1, p}(q)^{-1}\left(2 E_{1, p}(q)-K_{1, p}(q)\right), \\
& y(L)=\int_{0}^{L} \sin \theta(s) d s=0 .
\end{aligned}
$$

Proof. Changing the variable from $s$ to $\phi$ with (32), we have

$$
\begin{aligned}
x(L) & =\int_{0}^{L} \cos \theta(s) d s=m \int_{0}^{L / m} \cos \theta(s) d s \\
& =2 m q^{1-2 / p}\left(\frac{p-1}{2 p R}\right)^{1 / p} \int_{0}^{2 \pi} \frac{\cos \left\{2 \sin ^{-1}(q \sin \phi)\right\}|\cos \phi|^{1-2 / p}}{\sqrt{1-q^{2} \sin ^{2} \phi}} d \phi \\
& =2 m q^{1-2 / p}\left(\frac{p-1}{2 p R}\right)^{1 / p} \int_{0}^{2 \pi} \frac{1-2 q^{2} \sin ^{2} \phi}{\sqrt{1-q^{2} \sin ^{2} \phi}}|\cos \phi|^{1-2 / p} d \phi,
\end{aligned}
$$


where in the last equality, the relation

$$
\cos \left\{2 \sin ^{-1}(q \sin \phi)\right\}=1-2 \sin ^{2}\left(\sin ^{-1}(q \sin \phi)\right)=1-2 q^{2} \sin ^{2} \phi .
$$

was applied. Hence, using (33), we see that the right-hand-side of above equation becomes

$$
\begin{aligned}
& L\left(\int_{0}^{\pi / 2} \frac{(\cos \phi)^{1-2 / p}}{\sqrt{1-q^{2} \sin ^{2} \phi}} d \phi\right)^{-1} \int_{0}^{\pi / 2} \frac{1-2 q^{2} \sin ^{2} \phi}{\sqrt{1-q^{2} \sin ^{2} \phi}}|\cos \phi|^{1-2 / p} d \phi \\
& \quad=L\left(\int_{0}^{\pi / 2} \frac{(\cos \phi)^{1-2 / p}}{\sqrt{1-q^{2} \sin ^{2} \phi}} d \phi\right)^{-1} . \\
& \left(2 \int_{0}^{\pi / 2} \sqrt{\left.1-q^{2} \sin ^{2} \phi(\cos \phi)^{1-2 / p} d \phi-\int_{0}^{\pi / 2} \frac{(\cos \phi)^{1-2 / p}}{\sqrt{1-q^{2} \sin ^{2} \phi}} d \phi\right)}\right. \\
& =L K_{1, p}(q)^{-1}\left(2 E_{1, p}(q)-K_{1, p}(q)\right) .
\end{aligned}
$$

Next, we compute $y(L)$. By definition,

$$
\begin{aligned}
y(L) & =\int_{0}^{L} \sin \theta(s) d s=m \int_{0}^{L / m} \sin \theta(s) d s \\
& =2 m q^{1-2 / p}\left(\frac{p-1}{2 p R}\right)^{1 / p} \int_{0}^{2 \pi} \frac{\sin \left\{2 \sin ^{-1}(q \sin \phi)\right\}|\cos \phi|^{1-2 / p}}{\sqrt{1-q^{2} \sin ^{2} \phi}} d \phi .
\end{aligned}
$$

Noting the relation

$$
\int_{\pi}^{2 \pi} \frac{\sin \left\{2 \sin ^{-1}(q \sin \phi)\right\}|\cos \phi|^{1-2 / p}}{\sqrt{1-q^{2} \sin ^{2} \phi}} d \phi=-\int_{0}^{\pi} \frac{\sin \left\{2 \sin ^{-1}(q \sin \phi)\right\}|\cos \phi|^{1-2 / p}}{\sqrt{1-q^{2} \sin ^{2} \phi}} d \phi,
$$

we obtain the second assertion.

Here, we define the function $X_{1}$ as:

$$
X_{1}(q)=L K_{1, p}(q)^{-1}\left(2 E_{1, p}(q)-K_{1, p}(q)\right) .
$$

LeMma 2. For $X_{1}$, following properties hold.

(i) $X_{1}(q)$ is monotone decreasing on $(0,1)$.

(ii) $X_{1}(0)=L$.

(iii)

$$
X_{1}(1)= \begin{cases}-L & (1<p \leq 2) \\ -\frac{L}{p-1} & (2<p)\end{cases}
$$


Proof. Differentiating $K_{1, p}$ and $E_{1, p}$, with $q$ we have

$$
\begin{aligned}
& \frac{d K_{1, p}}{d q}(q)=q \int_{0}^{\pi / 2} \frac{(\sin \phi)^{2}(\cos \phi)^{1-2 / p}}{\left(1-q^{2} \sin ^{2} \phi\right)^{3 / 2}} d \phi>0 \\
& \frac{d E_{1, p}}{d q}(q)=-q \int_{0}^{\pi / 2} \frac{(\sin \phi)^{2}(\cos \phi)^{1-2 / p}}{\sqrt{1-q^{2} \sin ^{2} \phi}} d \phi<0 .
\end{aligned}
$$

So, $K_{1, p}(q)$ and $E_{1, p}(q)$ are monotone increasing and decreasing on $(0,1)$ respectively. Thus $X_{1}(q)=L\left(2 E_{1, p}(q) / K_{1, p}(q)-1\right)$ is monotone decreasing on $(0,1)$. For (ii), we note that

$$
E_{1, p}(0)=K_{1, p}(0)=\int_{0}^{\pi / 2}(\cos \phi)^{1-2 / p} d \phi=\frac{\sqrt{\pi} \Gamma\left(1-\frac{1}{p}\right)}{2 \Gamma\left(\frac{3}{2}-\frac{1}{p}\right)}<\infty,
$$

where $\Gamma(\cdot)$ is a Gamma function. From this, we obtain (ii). To see (iii), we note the relation

$$
\begin{aligned}
E_{1, p}(1) & =\int_{0}^{\pi / 2}(\cos \phi)^{2-2 / p} d \phi=\frac{\sqrt{\pi} \Gamma\left(\frac{3}{2}-\frac{1}{p}\right)}{2 \Gamma\left(2-\frac{1}{p}\right)} \\
\lim _{q \rightarrow 1} K_{1, p}(q) & =\int_{0}^{\pi / 2}(\cos \phi)^{-2 / p} d \phi= \begin{cases}\infty, & (1<p \leq 2) \\
\frac{\sqrt{\pi} \Gamma\left(\frac{1}{2}-\frac{1}{p}\right)}{2 \Gamma\left(1-\frac{1}{p}\right)}, & (2<p) .\end{cases}
\end{aligned}
$$

Thus, for the case $1<p \leq 2$ we have $X_{1}(1)=-L$. For the case $p>2$, we have from (37) and the formula $z \Gamma(z)=\Gamma(z+1)$ ([5, Formula 5.51]),

$$
X_{1}(1)=L\left(\frac{2 \Gamma\left(1-\frac{1}{p}\right) \Gamma\left(\frac{3}{2}-\frac{1}{p}\right)}{\Gamma\left(\frac{1}{2}-\frac{1}{p}\right) \Gamma\left(2-\frac{1}{p}\right)}-1\right)=L\left(\frac{2\left(\frac{1}{2}-\frac{1}{p}\right)}{\left(1-\frac{1}{p}\right)}-1\right)=-\frac{L}{p-1}
$$

From Lemma 2, it holds that $X_{1}(q)=a$ has a unique root for $(-L<a<L)$ if $(1<p \leq 2)$ and for $(-L /(p-1)<a<L)$ if $(2<p)$. Using this $q$ and (32), (33), we can express locus $(x, y)$ of $p$-elastic curve as 


$$
\begin{aligned}
x(s) & =\int_{0}^{s} \cos \theta(s) d s \\
& =\frac{L}{4 m}\left(K_{1, p}(q)\right)^{-1} \int_{0}^{\phi} \cos 2\left(\sin ^{-1}(q \sin \varphi)\right) \frac{|\cos \varphi|^{1-2 / p}}{\sqrt{1-q^{2} \sin ^{2} \varphi}} d \varphi \\
& =\frac{L}{4 m}\left(K_{1, p}(q)\right)^{-1} \int_{0}^{\phi}\left(1-2 q^{2} \sin ^{2} \varphi\right) \frac{|\cos \varphi|^{1-2 / p}}{\sqrt{1-q^{2} \sin ^{2} \varphi}} d \varphi=x(\phi) \\
y(s) & = \pm \int_{0}^{s} \sin \theta(s) d s \\
& = \pm \frac{L}{4 m}\left(K_{1, p}(q)\right)^{-1} \int_{0}^{\phi} \sin 2\left(\sin ^{-1}(q \sin \varphi)\right) \frac{|\cos \varphi|^{1-2 / p}}{\sqrt{1-q^{2} \sin ^{2} \varphi}} d \varphi=y(\phi) .
\end{aligned}
$$

Therefore, we have shown the case (ii) of Theorem 1 and 2. Especially, when $a=0$, locus $(x, y)$ makes a closed curve. This becomes like figure eight; see Figure 2-(vi). Thus we have proved the case (vi) of Theorem 1 and 2 .

The case (V) of (12).

As in the case (III) of (12), from (29), putting $q^{2}=(E+R) / 2 R$ and noting $\theta(0)=0, \theta(L)=2 n \pi$, we have

$$
\theta_{s}(s)=\left(\frac{2 p R}{p-1}\right)^{1 / p} \sqrt[p]{q^{2}-\sin ^{2} \frac{\theta}{2}}, \quad(0 \leq s \leq L)
$$

and hence

$$
\begin{aligned}
s & =\left(\frac{p-1}{2 p R}\right)^{1 / p} \int_{0}^{\theta} \frac{d \theta}{\sqrt[p]{q^{2}-\sin ^{2} \frac{\theta}{2}}}, \quad(0 \leq \theta \leq 2 n \pi) \\
& =\left(\frac{p-1}{p(E+R)}\right)^{1 / p} \int_{0}^{\theta} \frac{d \theta}{\sqrt[p]{1-r^{2} \sin ^{2} \frac{\theta}{2}}} \\
& =2\left(\frac{p-1}{p(E+R)}\right)^{1 / p} \int_{0}^{\phi} \frac{d \phi}{\sqrt[p]{1-r^{2} \sin ^{2} \phi}}, \quad(0 \leq \phi \leq n \pi),
\end{aligned}
$$

where $r=1 / q$ and $\phi=\theta / 2$. Thus it holds

$$
\frac{L}{2 n}=2\left(\frac{p-1}{p(E+R)}\right)^{1 / p} \int_{0}^{\pi / 2} \frac{d \phi}{\sqrt[p]{1-r^{2} \sin ^{2} \phi}},
$$


so, $R$ can be expressed as a function of $r$ :

$$
R(r)=R=\frac{2^{2 p-1} r^{2} n^{p}(p-1)}{p L^{p}}\left(\int_{0}^{\pi / 2} \frac{d \phi}{\sqrt[p]{1-r^{2} \sin ^{2} \phi}}\right)^{p} .
$$

Applying (40), we can express $(x(L), y(L))$ as followings:

LEMMA 3.

$$
\begin{aligned}
x(L) & =\int_{0}^{L} \cos \theta(s) d s=L\left(\int_{0}^{\pi / 2} \frac{d \phi}{\sqrt[p]{1-r^{2} \sin ^{2} \phi}}\right)^{-1}\left(\int_{0}^{\pi / 2} \frac{\cos 2 \phi}{\sqrt[p]{1-r^{2} \sin ^{2} \phi}} d \phi\right) \\
& =L K_{2, p}(r)^{-1}\left(\frac{r^{2}-2}{r^{2}} K_{2, p}(r)+\frac{2}{r^{2}} E_{2, p /(p-1)}(r)\right), \\
y(L) & =\int_{0}^{L} \sin \theta(s) d s=0 .
\end{aligned}
$$

Proof.

$$
\begin{aligned}
x(L) & =\int_{0}^{L} \cos \theta(s) d s=n \int_{0}^{L / n} \cos \theta(s) d s \\
& =4 n\left(\frac{p-1}{p(E+R)}\right)^{1 / p} \int_{0}^{\pi / 2} \frac{\cos 2 \phi}{\sqrt[p]{1-r^{2} \sin ^{2} \phi}} d \phi \\
& =L\left(\int_{0}^{\pi / 2} \frac{d \phi}{\sqrt[p]{1-r^{2} \sin ^{2} \phi}}\right)^{-1}\left(\int_{0}^{\pi / 2} \frac{\cos 2 \phi}{\sqrt[p]{1-r^{2} \sin ^{2} \phi}} d \phi\right) \\
& =L K_{2, p}(r)^{-1}\left(\frac{r^{2}-2}{r^{2}} K_{2, p}(r)+\frac{2}{r^{2}} E_{2, p /(p-1)}(r)\right)
\end{aligned}
$$

For $y(L)$, we have

$$
\begin{aligned}
y(L) & =\int_{0}^{L} \sin \theta(s) d s=n \int_{0}^{L / n} \sin \theta(s) d s \\
& =2 n\left(\frac{p-1}{p(E+R)}\right)^{1 / p} \int_{0}^{\pi} \frac{\sin 2 \phi}{\sqrt[p]{1-r^{2} \sin ^{2} \phi}} d \phi=0 .
\end{aligned}
$$

Here, we define the function $X_{2}$ as: 


$$
\begin{aligned}
X_{2}(r) & =L\left(\int_{0}^{\pi / 2} \frac{d \phi}{\sqrt[p]{1-r^{2} \sin ^{2} \phi}}\right)^{-1}\left(\int_{0}^{\pi / 2} \frac{\cos 2 \phi}{\sqrt[p]{1-r^{2} \sin ^{2} \phi}} d \phi\right) \\
& =L K_{2, p}(r)^{-1}\left(\frac{r^{2}-2}{r^{2}} K_{2, p}(r)+\frac{2}{r^{2}} E_{2, p /(p-1)}(r)\right) .
\end{aligned}
$$

For $X_{2}$, following properties hold.

\section{LEMMA 4.}

(i) $X_{2}(r)$ is monotone decreasing on $(0,1)$.

(ii) $X_{2}(0)=0$.

(iii)

$$
X_{2}(1)= \begin{cases}-L & (1<p \leq 2) \\ -\frac{L}{p-1} & (2<p) .\end{cases}
$$

Proof. Differentiating $K_{2, p}$ with respect to $r$ we have

$$
\frac{d K_{2, p}(r)}{d r}(r)=\frac{2 r}{p} \int_{0}^{\pi / 2}\left(1-r^{2} \sin ^{2} \phi\right)^{-1 / p-1} \sin ^{2} \phi d \phi>0,
$$

so $K_{2, p}(r)^{-1}$ is monotone decreasing. Moreover

$$
\begin{aligned}
& \frac{d}{d r}\left(\int_{0}^{\pi / 2} \frac{\cos 2 \phi}{\sqrt[p]{1-r^{2} \sin ^{2} \phi}} d \phi\right)=\frac{2 r}{p} \int_{0}^{\pi / 2} \cos 2 \phi(\sin \phi)^{2}\left(1-r^{2} \sin ^{2} \phi\right)^{-1 / p-1} d \phi \\
& =\frac{r}{p} \int_{-\pi / 2}^{\pi / 2}-\sin \varphi \sin ^{2}\left(\frac{\varphi}{2}+\frac{\pi}{4}\right)\left(1-r^{2} \sin ^{2}\left(\frac{\varphi}{2}+\frac{\pi}{4}\right)\right)^{-1 / p-1} d \varphi \\
& =\frac{r}{p} \int_{0}^{\pi / 2}-\sin \varphi\left\{\sin ^{2}\left(\frac{\varphi}{2}+\frac{\pi}{4}\right)\left(1-r^{2} \sin ^{2}\left(\frac{\varphi}{2}+\frac{\pi}{4}\right)\right)^{-1 / p-1}\right. \\
& \left.\quad-\sin ^{2}\left(-\frac{\varphi}{2}+\frac{\pi}{4}\right)\left(1-r^{2} \sin ^{2}\left(-\frac{\varphi}{2}+\frac{\pi}{4}\right)\right)^{-1 / p-1}\right\} d \varphi<0 .
\end{aligned}
$$

Thus, (i) is proved. (ii) is obvious. To see (iii), we note $E_{2, p /(p-1)}(1)=E_{1, p}(1)$ and $\lim _{r \rightarrow 1} K_{2, p}(r)=\lim _{q \rightarrow 1} K_{1, p}(q)$. From these relations, as in the case of $X_{1}(1)$, using (37) and formula $z \Gamma(z)=\Gamma(z+1)$, we obtain the result.

From Lemma 4, it holds that $X_{2}(r)=a$ has a unique root for $(-L<a<0)$ if $(1<p \leq 2)$ and for $(-L /(p-1)<a<0)$ if $(2<p)$. Using this $r$ and (39), (40), we can express locus $(x, y)$ of $p$-elastic curve as in the case (iii). Concrete expressions of $(x, y)$ are case (iii) of Theorem 1 and 2 . 
Next, from Figure 2-(iv), we can guess that the locus $(x, y)$ corresponding to $\alpha=\pi$ in (10), also becomes to a $p$-elastic curve. Indeed, if we take $\alpha=\pi$ in (10), instead of (38), we obtain

$$
\theta_{s}(s)=\left(\frac{2 p R}{p-1}\right) \sqrt[1 / p]{q^{2}-\cos ^{2} \frac{\theta}{2}}, \quad(0 \leq s \leq L) .
$$

Therefore locus $(x, y)$ generated by (44) becomes to case (iv) of Theorem 1 and 2. Similarly $x(L)$ and $y(L)$ can be expressed as

$$
\begin{aligned}
x(L) & =L\left(\int_{0}^{\pi / 2} \frac{d \phi}{\sqrt[p]{1-r^{2} \sin ^{2} \phi}}\right)^{-1}\left(\int_{0}^{\pi / 2} \frac{\cos 2 \phi}{\sqrt[p]{1-r^{2} \cos ^{2} \phi}} d \phi\right) \\
& =-L K_{2, p}(r)^{-1}\left(\int_{0}^{\pi / 2} \frac{\cos 2 \phi}{\sqrt[p]{1-r^{2} \sin ^{2} \phi}} d \phi\right) \\
& =-L K_{2, p}(r)^{-1}\left(\frac{r^{2}-2}{r^{2}} K_{2, p}(r)+\frac{2}{r^{2}} E_{2, p /(p-1)}(r)\right) .
\end{aligned}
$$

So, by Lemma $4, x(s)$ is monotone increasing and end values are $x(0)=0$, $x(L)=L$ if $(1<p \leq 2),(x(L)=L /(p-1)$ if $(p>2))$. For $y(L)$, we have

$$
y(L)=2 n\left(\frac{p-1}{p(E+R)}\right)^{1 / p} \int_{0}^{\pi} \frac{\sin 2 \phi}{\sqrt[p]{1-r^{2} \cos ^{2} \phi}} d \phi=0 .
$$

Finally, we consider the case (IV) of (12).

The case (IV) of (12).

This case corresponds to the case $q=1$ in the case (iii) and (iv) of Theorem 1 and 2. Assume $p \leq 2$, then we have from (39)

$$
\lim _{\phi \rightarrow \pi / 2} s(\phi)=2\left(\frac{p-1}{2 p R}\right)^{1 / p} \int_{0}^{\pi / 2}(\cos \phi)^{-2 / p} d \phi=\infty .
$$

Hence we do not have any $p$-elastic curve of finite arc-length. But, in the case $p>2$ from (37), above limit is finite. So, we can expect the existence of $p$-elastic curve in this case. We see that from (22), $F^{ \pm}(l)$ and from (20), $F L^{ \pm}(l), F R^{ \pm}(l)$ are obtained. Indeed, appropriate combinations of $F^{ \pm}(l), F L^{ \pm}(l), F R^{ \pm}(l)$ and line segments are as Theorem 3 stationary curves ( $p$-elastic curves). We will show this by an example.

Example 1. $F^{+}\left(L_{0}\right) L\left(L_{1}\right) F^{+}\left(L_{0}\right) L\left(L_{2}\right)$ is a stationary curve of $J(\theta)$ under the condition $\theta \in W(p, a, 2)$, where $a=2 L_{0} /(p-1)+L_{1}+L_{2}$. 


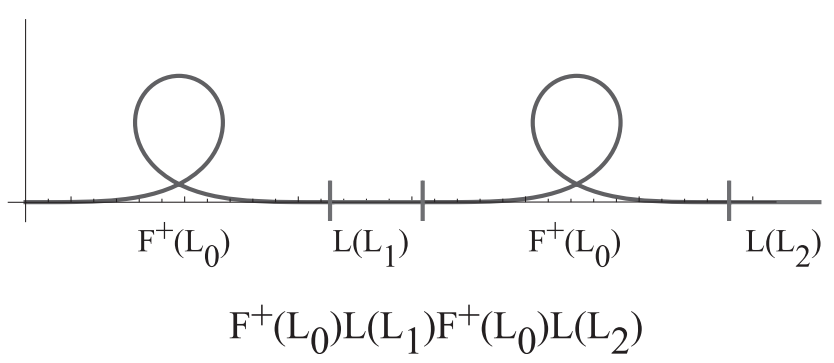

FigURE 6. $\quad F^{+}\left(L_{0}\right) L\left(L_{1}\right) F^{+}\left(L_{0}\right) L\left(L_{2}\right)$.

We note that $\theta_{s s}$ is discontinuous at $s=L_{0}, L_{0}+L_{1}, 2 L_{0}+L_{1}$, so we divide the integration of first variation of $J$ with these points. Noting $\theta_{s}(s)=0$ at $s=L_{0}, L_{0}+L_{1}, 2 L_{0}+L_{1}$, and $R$ is given by (41), we have

$$
\begin{aligned}
J^{\prime}(\theta)[\varphi]= & \int_{0}^{L_{0}}\left|\theta_{s}(s)\right|^{p-2} \theta_{s}(s) \varphi_{s}(s) d s+\int_{L_{0}}^{L_{0}+L_{1}} \cdot d s+\int_{L_{0}+L_{1}}^{2 L_{0}+L_{1}} \cdot d s+\int_{2 L_{0}+L_{1}}^{L} \cdot d s \\
= & {\left[\left|\theta_{s}\right|^{p-2} \theta_{s} \varphi\right]_{0}^{L_{0}}+[\cdot]_{L_{0}}^{L_{0}+L_{1}}+[\cdot]_{L_{0}+L_{1}}^{2 L_{0}+L_{1}}+[\cdot]_{2 L_{0}+L_{1}}^{L} \int_{L_{0}}^{2 L_{0}+L_{1}} \cdot d s-\int_{L_{0}+L_{1}}^{L} \cdot d s } \\
& -\int_{0}^{L_{0}}\left(\left|\theta_{s}(s)\right|^{p-2} \theta_{s}(s)\right)_{s} \varphi(s) d s-\int_{L_{0}+L_{1}}^{L_{0}+L_{1}} \cdot d s-\int_{L_{0}}^{L_{0}} R \sin (2 \pi) \varphi(s) d s \\
= & \int_{0}^{L_{0}} R \sin \theta(s) \varphi(s) d s+\int_{2 L_{0}+L_{1}}^{L_{0}+L_{1}} R \sin (4 \pi) \varphi(s) d s \\
& +\int_{L_{0}+L_{1}}^{2 L_{0}+L_{1}} R \sin \theta(s) \varphi(s) d s+\int_{0}^{L} R \sin \theta(s) \varphi(s) d s=0, \\
= & \int_{0}^{L} R
\end{aligned}
$$

where the last equality holds from (8). We can see that the curve in the cases (vii), (viii) and (ix) of Theorem 3 are stationary by the same reason. Finally, we show that in this case $p$-elastic curve exists for $a$ satisfying $L /(p-1) \leq a<L$ (case (vii)), and $-L<a \leq-L /(p-1)$ (cases (viii) and (ix)). For the case (vii), we have

$$
a=\frac{L_{0}}{p-1} n+\sum_{i=1}^{n_{0}} L_{i} .
$$

On the other hand, we know the relation $n L_{0}+\sum_{i=1}^{n_{0}} L_{i}=L$. Eliminating $L_{0}$ from (45), we obtain

$$
a=\frac{L+(p-2) \sum_{i=1}^{n_{0}} L_{i}}{p-1} .
$$


The cases (viii) and (ix) are shown similarly. Now, we have finished the proofs of Theorem 1-3.

\section{Appendix}

Let us define

$$
\begin{aligned}
W_{0}(p, a, n):= & \left\{\psi \in W_{0}^{1, p}(0, L) \mid \int_{0}^{L} \cos \left(\psi(s)+\frac{2 n \pi}{L} s\right) d s=a,\right. \\
& \left.\int_{0}^{L} \sin \left(\psi(s)+\frac{2 n \pi}{L} s\right) d s=0\right\} .
\end{aligned}
$$

We note, for $\psi \in W_{0}^{1, p}(0, L)$ it holds that (by integration by parts)

$$
\left(\int_{0}^{L}|\psi(s)|^{p} d s\right)^{1 / p} \leq L\left(\int_{0}^{L}\left|\psi_{s}(s)\right|^{p} d s\right)^{1 / p}
$$

Thus we can assume $W_{0}^{1, p}(0, L)$ has a norm of the form

$$
\|\psi\|_{W_{0}^{1, p}(0, L)}=\left(\int_{0}^{L}\left|\psi_{s}(s)\right|^{p} d s\right)^{1 / p}
$$

Lemma 5. $\inf _{\theta \in W_{0}(p, a, n)} J(\theta)$ is attained.

Proof. Applying $A=\psi_{s}+2 n \pi / L, B=-2 n \pi / L$ to

$$
\left(\frac{|A|^{p}+|B|^{p}}{2}\right) \geq\left(\frac{|A|+|B|}{2}\right)^{p} \geq\left(\frac{|A+B|}{2}\right)^{p}
$$

and putting $\psi=\theta-2 n \pi s / L$, where $\theta \in W(p, a, n)$ we have

$$
\begin{aligned}
J(\theta) & =J\left(\psi+\frac{2 n \pi s}{L}\right)=\frac{1}{2} \int_{0}^{L}\left|\psi_{s}+\frac{2 n \pi}{L}\right|^{p} d s \\
& \geq 2^{-p} \int_{0}^{L}\left|\psi_{s}\right|^{p} d s-\frac{L}{2}\left(\frac{2 n \pi}{L}\right)^{p} .
\end{aligned}
$$

Thus we can assume $\|\psi\|_{W_{0}^{1, p}(0, L)}$ is bounded. Let us show that $W_{0}(p, a, n)$ is weakly closed. Let $\left\{\psi_{m}\right\} \subset W_{0}(p, a, n)$ and $\psi_{m} \rightarrow \psi_{0}$. Since $W_{0}^{1, p}(0, L)$ is compactly embedded in $C[0, L]\left(\left[2\right.\right.$, p. 212]), if $\psi_{m} \rightarrow \psi_{0}$, then $\psi_{0} \in W_{0}(p, a, n)$. Thus $W_{0}(p, a, n)$ is weakly closed, and hence $W_{0}(p, a, n) \cap\left\{\psi \in W_{0}^{1, p}(0, L) \mid\right.$ $\left.\|\psi\|_{W_{0}^{1, p}(0, L)} \leq C\right\}$ is weakly compact, where $C>0$ is sufficientlt large. Noting that $J(\psi+2 n \pi s / L)$ is (weakly) lower-semi-continuous on $W_{0}^{1, p}(0, L)$, we obtain the result. 


\section{Acknowledgement}

The auther expresses his hearty thanks to anonymous refree for many valuable suggestions.

\section{REFERENCES}

[1] S. S. Antman, Nonlinear problems of elasticity, Springer, New York, 1995.

[2] H. Brezis, Functional analysis, Sobolev spaces and partial differential equations, Springer, New York, 2011.

[3] N. KoIso, Variational problem, Kyoritsu Syuppan, Tokyo, 1998.

[4] W. Matsumoto, M. Murai and S. Yotsutani, By which kind of sound can one hear the shape of a drum?, RIMS kôkyûroku 1315 (2003), 156-175.

[5] F. Olver, D. Lozier, R. Boisvert and C. Clark, NIST handbook of mathematical functions, Cambridge Univ. Press, New York, 2010.

[6] S. TAKeuchi, Generalized Jacobian elliptic functions and their application to bifurcation problems associated with $p$-Laplacian, J. Math. Anal. Appl. 385 (2012), 24-35.

[ 7] S. TAKeuchI, Generalized Jacobian elliptic functions, RIMS kôkyûroku 1838 (2012), 71-101.

[8] C. Truesdell, The influence of elasticity on analysis: the classic heritage, Bull. Amer. Math. Soc. 9 (1983), 293-310.

[9] H. Yanamoto, On the elastic closed curves, Kodai Math. J. 8 (1985), 224-235.

[10] K. Watanabe, Plane domains which are spectrally determined, Ann. Global Anal. and Geom. 18 (2000), 447-475.

Kohtaro Watanabe

Department of Computer Science

National Defense Academy

1-10-20 HashirimizU

YoKOSUKA 239-8686

JAPAN

E-mail: wata@nda.ac.jp 